\title{
DEVELOPMENT OF AUTONOMOUS BLIMP ROBOT WITH INTELLIGENT CONTROL
}

\author{
Keiko Motoyama, Hidenori Kawamura, \\ Masahito Yamamoto and Azuma Ohuchi \\ Graduate School of Engineering, Hokkaido University
}

\begin{abstract}
The purpose of this study is to realize an autonomous robotic blimp. While this robot can pass over terrain that may be impossible for landbased robots to negotiate, it is hard to control autonomously because it is subjected to strong inertial forces and air resistance. We are now designing an autonomously controlled indoor blimp, which will be suitable for use in for inspections or monitoring tasks and as an amusement robot or guide robot.
\end{abstract}

Keywords: Blimp control, Autonomous robot

\section{Introduction}

The purpose of this study is to realize an autonomous robotic airship, also known as a blimp or lighter-than-air vehicle. Studies on the utilization of unmanned aerial vehicles have recently grown with an increasing focus on blimps, because there are many advantages to using a blimp. First, a flying robot can pass over terrain that may be impossible for land-based robots to negotiate, so we need not consider the state of a floor or the existence of stairs. Second, as a blimp outperforms airplanes and helicopters at low speed and low altitude, it is suitable for use in inspections or monitoring tasks. Furthermore, a blimp needs no energy to maintain an altitude, as it relies on its neutral buoyancy to stay aloft.

A robot like an automatically controlled blimp could be of great use in various areas. It could be used for indoor observation in a disaster, such as after an earthquake, and for inspections in high places, etc. Also, high-tech amusement robots, such as AIBO or ASIMO, have attracted attention recently. It is possible for a blimp to become a 3D amusement robot by, for example, communicating with people or making entertaining movements in an advertisement. Moreover, a blimp could act as a kind of guide robot. Thus, indoor blimps can be used in a variety of applications.

The original version of this chapter was revised: The copyright line was incorrect. This has been corrected. The Erratum to this chapter is available at DOI: 10.1007/978-0-387-35660-0_65 
However, it is very difficult to control a blimp autonomously for following reasons.

- A blimp needs three-dimensional position and attitude control.

- A blimp's payload is restricted. Therefore, a blimp's control system must be based on a few light sensors, like noisy sonar data.

- A blimp has great inertia and unstable dynamics and is greatly affected by temperature and atmospheric pressure. The navigation methods for a blimp must cope with the unpredictable forces of wind and air gusts.

In order to cope with a dynamic and unknown environment, one of the possible solutions is use of a learning algorithm, by which a blimp acquires specific patterns of behavior. With learning, it will be able to function even if one of its propellers breaks down. This control method technology for a blimp could be applied to other robots, especially submarine or space robots. In this paper, we state a dynamic model for a blimp and construct a blimp simulator. This simulator enables lowcost development and system testing before implementation in an actual blimp robot.

\section{Related Works}

Blimps have been studied in various contexts, including some experimental work. Wyeth [7] describes the design of a lighter-than-air autonomous robot and its natural landmark dimensions. The robot was programmed to fly a course by giving the robot a sequence of landmarks and trajectories, and has successfully flown.

A method for station keeping and docking of a lighter-than-air vehicle based on visual input is given in [6]. A small-size indoor blimp was used, equipped with an on-board camera, radio control and video link. In addition, they proposed an image-based control law. Image-based control of an indoor blimp is also addressed in [8].

Ramos et al. present one of the Internet-accessible airship simulators, based on a comprehensive airship dynamic model [5]. The simulator is meant to be used as a tool for the development of control and navigation methods for semi-autonomous robotic airships and as a test-bed for airship pilot training. This project's goal is to provide a low-speed, low-altitude aerial vehicle for environmental monitoring missions.

In these studies, blimps are controlled by systems based on a PID controller. However, it is difficult to cope with unpredictable forces like wind using such a fixed controller. So we use a combination of a basic control method and a learning or genetic algorithm. 


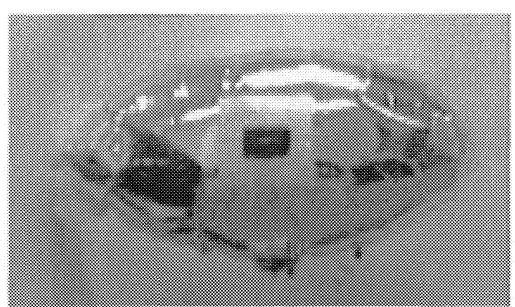

Figure 1. Micro-blimp

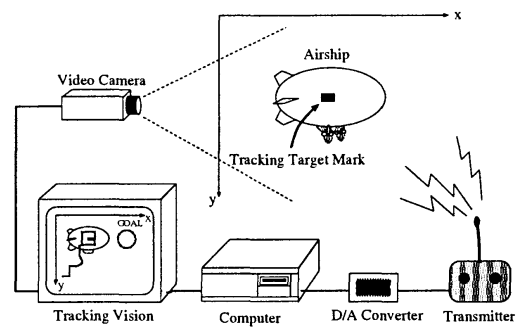

Figure 2. Micro-blimp Control System

\section{Previous Study}

A blimp is hard to control because it has large inertia and has to move by predicting the next action. As prediction depends on the characteristics of each blimp and spatial conditions, a fixed control method cannot cope with those changes. In order to acquire adaptive control of a blimp in continuous environments, a combination of CMAC-based Q-learning and adaptive construction of a state space with GA was proposed in our previous study [3]. The CMAC-based Q-learning algorithm (Q-CMAC) [4] is a Q-learning algorithm using CMAC as an approach to the generalization problem.

It is difficult for a blimp to learn in the real world, because the state space segmentation method proposed for that environment requires many trials using GA and reinforcement learning. The state space is therefore constructed in a simulated environment, then the learning method is applied to a real micro blimp, based on the state space segmentation acquired in a simulated environment.

A micro-blimp (Figure 1), nearly $1 \mathrm{~m}$ long and $0.5 \mathrm{~m}$ wide, was used in our previous study because it is suitable to be tested in a laboratory environment. This micro-blimp does not have enough payload capacity to carry sensory equipment and whole control units. Therefore, control commands are sent to the blimp by a radio transmitter. A receiver on the blimp turns two propellers for horizontal movements and one propeller for vertical movements. Also, the status of the blimp must be observed from external sensors. We use a tracking vision system with one CCD camera as external sensors. Therefore, our experimental sensing area for a blimp is restricted in two-dimensional vertical space. Figure 2 shows a blimp control system. A tracking vision system traces a mark on the blimp and produces its position and the velocity, which is projected into a two-dimensional vertical area. 
The control task is to pass two sub-goals in a specific order and reach a final goal without flying over the restricted vertical vision area. To accomplish a given task, a learning system gets a position, a velocity, a relative distance and the direction to a sub-goal or final goal. According to the given sensory information, the learning system must select the appropriate action from nine actions, which is a combination of horizontal movement (forward, backward and stop) and vertical movement (upward, downward and stop).

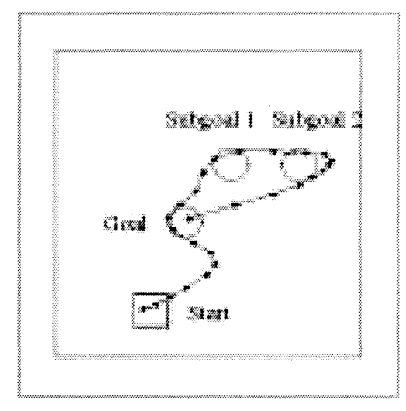

Figure 3. Successful track

Figure 3 shows an example of a successful track. In a simulation, we approximated the movement characteristics of a blimp, but changes in buoyancy due to differences in temperature and reduction in output of voltage were not taken into account. In the learning experiments with actual systems, batteries were changed several times and the characteristics of the blimp were altered during a process. Even if such noises were included, the actual system based on evolutionary segmentation could achieve a given task.

\section{Development of New Blimp Robot}

We are now constructing an autonomous control system for a new blimp, which is $3.5 \mathrm{~m}$ long and $2 \mathrm{~m}$ wide as shown in Figure 4 . In contrast to our previous micro blimp, this blimp has nearly $1.2 \mathrm{~kg}$ lift remaining for batteries, computers and sensors. We must select components which are as light as possible. The controller is based around the $\mathrm{H} 8 / 3048$ microcomputer. This is a 16 bit $20 \mathrm{MHz}$ microcomputer featuring: 5 PWM channels, 8 channels 10 bit A/D conversion and serial communications. A wireless unit is also needed to monitor the operations of a computer. A 3D motion sensor and sonar sensors will be used as internal sensors. We plan to load an on-board camera in the future. 


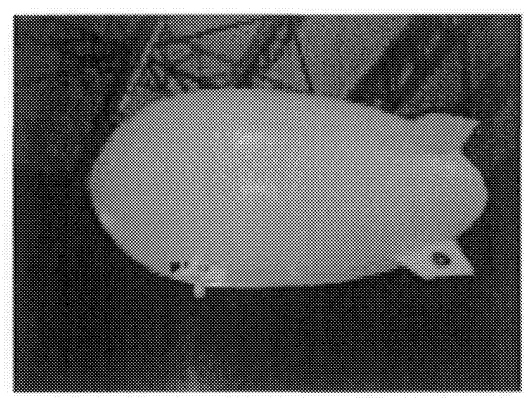

Figure 4. Blimp

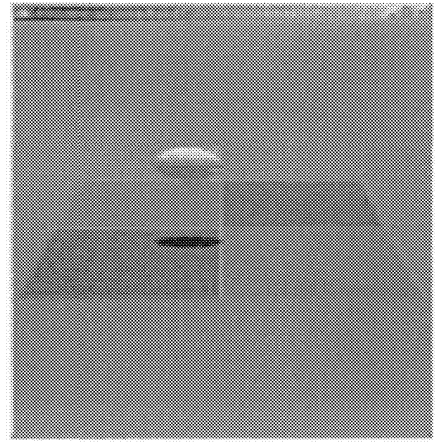

Figure 5. Simulator

The control system has to deal with a multi-input multi-output control problem, operating in a non-linear fashion with significant unpredictable disturbances. It will be effective to use a basic control method, like a PID controller in combination with a learning or genetic algorithm.

As we demonstrated in our previous study, use of a simulator is effective for decreasing temporal and spatial costs. A 3D simulator is consequently constructed for this blimp.

\section{Dynamics of the blimp}

A full six degrees-of-freedom mathematical model for this blimp is derived based on [2]. The blimp's center of mass changes during flight, so motion has to be referenced to by a system of orthogonal body axes placed at the center of volume (CV), as shown in Figure 6. The CV is also assumed to coincide with the center of buoyancy.

Considering the blimp to be a rigid body (aeroelastic effects can be ignored) in ideal fluid, and the airframe to be symmetric about X-Z (vertical) plane, the dynamic model can be stated as [2], [6].

$$
M \dot{x}+C(x)+D(x) x+G(\alpha, \beta, \gamma)=\tau,
$$

where $x=\left[\begin{array}{llllll}u & v & w & p & q & r\end{array}\right]^{T}$ is the velocity vector containing the three components of linear velocity $u, v, w$ and the three angular velocities $p, q, r$, all written with respect to the body-fixed reference frame XYZ. $M$ is the mass matrix containing all masses and inertias of the blimp as well as added mass and inertia terms. $C$ is the dynamic force vector containing the Coriolis and centrifugal terms of the dynamic model. $D(x)$ is the aerodynamic damping matrix containing linear and quadratic skin friction drag and damping due to vortex shedding, which depends on 

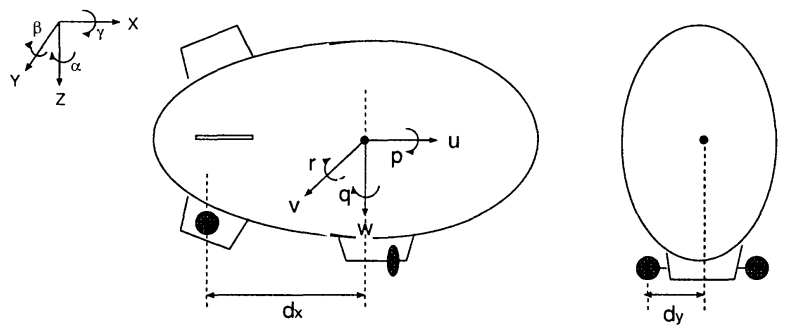

Figure 6. Reference frames

the streamline of the airship's hull. $G$ is the restoring forces vector containing the gravity and buoyancy forces resolved into body-fixes axes by a rotation matrix, parameterized by the standard $\operatorname{roll}(\gamma), \operatorname{pitch}(\beta)$ and yaw $(\alpha)$ angles representation. $\tau$ is the applied forces vector containing all forces and moments acting on the rigid body due to the aerodynamic control surfaces and the propulsive units. For an indoor blimp moving at low speed under laminar boundary layer conditions, aerodynamic control surfaces will have no effect and thus are not included. $\tau=\left[\begin{array}{lllllll}T_{c m n} & 0 & T_{v} & 0 & \left(d_{z} T_{c m n}\right) & \left(d_{y} T_{d i f f}\right)\end{array}\right]^{T}$, where $d_{y}$ is the horizontal offset from the center of volume of the horizontal propellers and $d_{x}$ is the vertical offset of the tail propeller, as shown in Figure 6.

\subsection{Linearized equations of motion}

Linearizing about $\beta=\gamma=0$ is a reasonable condition for blimp operation. Assuming blimp motion to be constrained to small perturbations about some equilibrium condition, a considerably simplified linear model can be obtained. The dynamic model system becomes decoupled in longitudinal motion and lateral motion [6].

The longitudinal or vertical model describes the airship motion in the $\mathrm{X}-\mathrm{Z}$ plane of the body-fixed reference frame. The corresponding state and input vectors are given by:

$$
x=\left[\begin{array}{llll}
\delta u & \delta w & \delta q & \delta \beta
\end{array}\right]^{T}, \quad \tau=\left[\begin{array}{ll}
\delta T_{f} & \delta T_{v}
\end{array}\right]^{T},
$$

where the small perturbation of the pitch angle, $\delta \beta$, is included in the state vector to accommodate the gravitation and buoyancy forces so as to obtain squared matrices. $T_{f}$ is the resultant force of thrust coming from two propellers on a gondola and $T_{v}$ is the thrust from a vertical propeller. The linearized longitudinal model about $\beta=\gamma=0$ is given by: 


$$
\begin{aligned}
M \dot{x}= & A x+B \tau+F, \\
M= & {\left[\begin{array}{cccc}
m+A_{11} & 0 & m a_{z} & 0 \\
0 & m+A_{33} & -m a_{x} & 0 \\
m a_{z} & -m a_{x} & I_{y}+A_{55} & 0 \\
0 & 0 & 0 & 1
\end{array}\right], } \\
A= & {\left[\begin{array}{cccc}
-X_{u} & 0 & 0 & -\left(m g-f_{b}\right) \\
0 & -Z_{w} & 0 & 0 \\
0 & 0 & -M_{q} & -a_{z} m g \\
0 & 0 & 1 & 0
\end{array}\right], } \\
B= & {\left[\begin{array}{llll}
1 & 0 & d_{z} & 0 \\
0 & 1 & 0 & 0
\end{array}\right]^{T}, F=\left[\begin{array}{c}
\left(m+A_{22}\right) r v+m a_{x} r^{2} \\
0 \\
\left(I_{y}+A_{55}\right) r^{2}-a_{x} r v \\
0
\end{array}\right] }
\end{aligned}
$$

$F$ is the linearized dynamic force vector containing the Coriolis and centrifugal terms about $\beta=\gamma=0$.

The lateral or horizontal model describes the dynamics of the airship orientation in the X-Y plane and includes the following state and input variables:

$$
x=\left[\begin{array}{llll}
\delta v & \delta p & \delta r & \delta \gamma
\end{array}\right]^{T}, \quad \tau=\delta T_{\text {rot }},
$$

where $T_{\text {rot }}$ is the thrust from the rear propeller for rotation. Here the roll angle, $\gamma$, is included and the linearized model is given by:

$$
\begin{aligned}
M \dot{x}= & A x+B \tau+F, \\
M= & {\left[\begin{array}{cccc}
m+A_{22} & -m a_{z} & m a_{x} & 0 \\
-m a_{z} & I_{x}+A_{44} & 0 & 0 \\
m a_{x} & 0 & I_{z}+A_{66} & 0 \\
0 & 0 & 0 & 1
\end{array}\right], } \\
A= & {\left[\begin{array}{cccc}
-Y_{v} & 0 & 0 & m g-f_{b} \\
0 & -K_{p} & 0 & -a_{z} m g \\
0 & 0 & -N_{r} & 0 \\
0 & 0 & 1 & 0
\end{array}\right], } \\
B= & {\left[\begin{array}{llll}
0 & 0 & d_{x} & 0
\end{array}\right]^{T}, F=\left[\begin{array}{c}
-\left(m+A_{11}\right) u r \\
m a_{x} u r \\
-m a_{x} u r \\
0
\end{array}\right] }
\end{aligned}
$$

$m$ is the blimp mass, $g$ is the gravitational acceleration and $f_{b}$ is the buoyancy force. Other parameters are shown in Table 1. Added mass and inertia terms $\left(A_{11} \sim A_{66}\right)$ are calculated on the assumption that the blimp is ellipsoid. The linear friction terms $\left(X_{u}, Y_{v}, Z_{w}, K_{p}, M_{q}, N_{r}\right)$ can 
be obtained by a wind-tunnel test or aerodynamic stability derivatives. They are calculated here based on an equation to calculate coefficients for a forward motion $\left(X_{u}\right)$ of a big airship [1]. As the blimp used in this study is small and can rotate on the spot, we adjust the value of other friction terms so as to adapt them to our blimp.

Table 1. Parameters

\begin{tabular}{lcc}
\hline added mass for $\mathrm{X}, \mathrm{Y}, \mathrm{Z}$ axes & $A_{11}, A_{22}, A_{33}$ & $1.32,4.537,4.537$ \\
\hline added inertia terms & $A_{44}, A_{55}, A_{66}$ & $0.0,1.252,1.252$ \\
\hline coordinates of the center of mass & $a_{x}, a_{z}$ & $-0.034,0.287$ \\
\hline coordinates of the propellers & $d_{x}, d_{z}$ & $1.36,0.95$ \\
\hline moments of inertia about $\mathrm{X}, \mathrm{Y}, \mathrm{Z}$ axes & $I_{x}, I_{y}, I_{z}$ & $2.833,4.75,3.684$ \\
\hline linear friction terms & $X_{u}, Y_{v}, Z_{w}$ & $0.118853,2.262096,2.262096$ \\
\hline linear friction terms & $K_{p}, M_{q}, N_{r}$ & 0.0594265 \\
\hline
\end{tabular}

\section{Conclusion}

In this paper, we state a dynamic model for a blimp and construct a blimp simulator. This simulator allows for low-cost development and system testing before implementation in the actual robot. Our ongoing work is focused on developing more robust and adequate control methods and implementing them in an actual blimp robot.

\section{References}

[1] Akira Azuma. Aeronautical Engineering(I) (in Japanese). Syoukabo, Tokyo, 1989.

[2] Sergio B. Varella Gomes and Josue G Ramos Jr. Airship dynamic modeling for autonomous operation. Proceedings of the IEEE International Conference on Robotics and Automation, pages 3462-3467, 1998.

[3] Keiko Motoyama et al. Evolutionary design of learning state space for small airship control. Proceedings of the Sixth International Conference on Simulation of Adaptive Behavior, pages 307-316, 2000.

[4] N. Ono and Y. Fukuta. Learning to coordinate in a continuous environment. ICMAS'96 Workshop Notes on Learning, Interactions and Organizations in Multiagent Environment., 1996.

[5] Josue G Ramos Jr., Silvio M. Maeta, et al. Development of VRML/JAVA unmanned airship simulating. IEEE/RSJ International Conference on Intelligent Robots and Systems, pages 1354-1359, 1999.

[6] Jose Santos-Victor Sjored van der Zwaan, Alexandre Bernardino. Vision based station keeping and docking for an aerial blimp. IEEE/RSJ International Conference on Intelligent Robots ans Systems, 2000.

[7] Gordon Wyeth and Ivan Barron. An Autonomous Blimp, pages 464-470. Springer Verlag, 1998.

[8] Hong Zhang and James P. Ostrowski. Visual servoing with dynamics: Control of an unmanned blimp. Proceedengs of IEEE Conference of Robotics and Automation, 1999. 